\title{
A Practical Approach to Differential Diagnosis of Cardiomyopathies with Infiltrative Phenotypes
}

\author{
Ligia Lopes Balsalobre Trevizan ${ }^{1 \oplus}$ and Sandrigo Mangini, ${ }^{1,2} \odot$ \\ Instituição: Hospital Israelita Albert Einstein, ${ }^{1}$ São Paulo, SP - Brazil \\ Instituto do Coração, ${ }^{2}$ São Paulo, SP - Brazil
}

\section{Introduction}

Heart failure (HF) affects approximately $2 \%$ of the world population; heart failure with preserved ejection fraction (HFpEF) is responsible for approximately $50 \%$ of cases, and it has shown a progressive increase in prevalence with the aging of the population. ${ }^{1}$

HFpEF is associated with high morbidity and mortality, and it is responsible for frequent hospitalizations, especially in the most elderly population. It has a complex pathophysiology and a diversified group of risk factors and etiologies. To date, few therapies have been shown to be effective in reducing cardiovascular outcomes in HFpEF; however, for specific etiologies, the possibility of disease-modifying therapeutic strategies exists. ${ }^{2-4}$

Restrictive cardiomyopathies (RCM) represent a small yet significant portion of patients with HFpEF, and they are characterized by diastolic dysfunction secondary to myocardial infiltration or primary dysfunction due to ventricular hypertrophy. RCM can result from hereditary or acquired diseases or a combination of both. Depending on their etiology, RCM are classified as infiltrative, non-infiltrative, storage diseases, and endomyocardial, and they may vary by age group. Some are more prevalent in certain regions (Table 1 ).

The most common phenotypic characteristic of infiltrative RCM is increased ventricular wall thickness; amyloidosis and storage diseases are the main etiologies, and situations of hypertrophy (hypertensive cardiomyopathy, aortic stenosis, hypertrophic cardiomyopathy, and their phenocopies) are differential diagnoses (Figure 1). Due to the heterogeneous nature and variable phenotypic expression of RCM, diagnosis is a challenge; however, it plays a prognostic and therapeutic role. Early recognition remains a fundamental barrier to having an impact on survival in these cases. ${ }^{5-7}$ The objective of this publication is to assist clinicians in reasoning by means of a practical approach to differential diagnosis of RCM with infiltrative phenotypes.

\section{Pathophysiology}

Ventricular walls evolve with increased stiffness and reduced relaxation, resulting in impaired filling and consequent increase

\section{Keywords}

Amyloidosis; Heart Failure; Cardiomyopathy, Restrictive

Mailing Address: Sandrigo Mangini •

Rua Lúcio Martins Rodrigues, 330 ap 13, Jardim Leonor Postal code 05621 025 São Paulo - SP

E-mail: sandrigomangini@cardiol.br, sandrigoman@uol.com.br

Manuscript received September 22, 2021, revised manuscript October 11, 2021, accepted October11, 2021.

DOI: https://doi.org/10.36660/abchf.20210036 in pressure while resting and, more intensely, during exertion; this severe diastolic dysfunction results in limited increases in end-diastolic volume of one or both ventricles. In addition, noncompliant ventricles inhibit rapid venous return, resulting in limited increases in stroke volume. Biatrial enlargement is characteristic; systolic function is generally preserved or slightly reduced, as is the size of the ventricular chambers, until advanced stages of the disease occur. ${ }^{5,7}$

RCM do not have a uniformly accepted diagnostic criterion; additionally, the classic pattern of restriction can be found in other diseases that affect the heart. In a simultaneous hemodynamic study of the ventricles, initial diastolic pressure declines and rises rapidly, with a tendency to equalize end-diastolic pressures of both ventricles. This restrictive physiology has been attributed to a reduction in ventricular compliance secondary to fibrosis or an infiltrative process in the subendocardium and/or myocardium. In $\mathrm{RCM}$, right atrial pressure usually exceeds $15 \mathrm{mmHg}$, and there should be a difference of at least $5 \mathrm{mmHg}$ between right atrial pressure and pulmonary capillary pressure, as well as between the end-diastolic pressures of the left and right ventricles, due to the unequal involvement and compliance of both ventricles, unlike what is found in constrictive pericarditis, where equalization of ventricular filling pressures occurs. ${ }^{8}$

\section{Clinical characteristics}

$\mathrm{HF}$ is a common initial manifestation, and intolerance to physical exertion is a common complaint. Arrhythmias and conduction disturbances are often found due to progressive biatrial enlargement. Physical examination may reveal findings of pulmonary congestion and, especially, systemic congestion, including jugular stasis, hepatomegaly, ascites, lower limb edema, and the presence of B3 and B4; Kussmaul's sign is usually absent. Mitral and tricuspid regurgitation may be present. ${ }^{9}$ Thromboembolic complications, with or without concomitant atrial fibrillation, are not uncommon due to marked biatrial enlargement and low atrial contractility. ${ }^{10}$ The clinical phenotype of HF due to systemic diseases that affect the heart is very similar; however, extracardiac manifestations are varied, and they increase diagnostic suspicion of specific etiologies, in addition to family history of heart disease (Table 2).

\section{Diagnostic investigation (Figure 2)}

\section{Laboratory exams}

Troponin and NT-proBNP may be elevated, and they have prognostic value. Renal function can be altered when there is renal infiltration or in patients with advanced HF. When amyloidosis is suspected, free light chain assay and serum and urinary immunofixation have high sensitivity for diagnosing light chain amyloidosis (AL). 


\section{Viewpoint}

\begin{tabular}{|c|c|c|c|c|}
\hline $\begin{array}{l}\text { Involvement of the } \\
\text { myocardium }\end{array}$ & $<30$ years & $\begin{array}{c}\text { Age } \\
30 \text { to } 65 \text { years }\end{array}$ & $>65$ years & Genetic \\
\hline \multirow[t]{4}{*}{ Non-infiltrative } & Idiopathic & Idiopathic & Idiopathic & Acquired \\
\hline & Scleroderma & Scleroderma & & Acquired \\
\hline & & Elastic pseudoxanthoma & & ABCC6 \\
\hline & & & Diabetic cardiomyopathy & Acquired \\
\hline \multicolumn{5}{|l|}{ Infiltrative } \\
\hline & & ATTR Sarcoidosis & & TTR gene variants* \\
\hline & & & ATTR-WT** & Acquired \\
\hline & & & AL amyloidosis & Acquired \\
\hline & Sarcoidosis & Sarcoidosis & & Acquired \\
\hline \multicolumn{5}{|l|}{ Storage diseases } \\
\hline & Hemochromatosis & & & HAMP, HFE, HFE2, HJV, PNPLA3, SLC40A1, TfR2 \\
\hline & Fabry disease & & & GLA \\
\hline & Gaucher disease & & & GBA \\
\hline & Hurler disease & & & IDUA \\
\hline & Glycogen deposition ${ }^{* * *}$ & & & Due to a specific type \\
\hline & & Iron overload & Iron overload & Acquired \\
\hline \multicolumn{5}{|l|}{ Endomyocardial } \\
\hline & Endomyocardial fibrosis & & & Acquired \\
\hline & Eosinophilic syndrome & Eosinophilic syndrome & Eosinophilic syndrome & Acquired \\
\hline & & Carcinoid disease & & Acquired \\
\hline & & & Metastatic tumor & Acquired \\
\hline & & & Radiation & Acquired \\
\hline & & Medication-related & Medication-related & Acquired \\
\hline
\end{tabular}

AL: light chain; ATTR: transthyretin amyloidosis; ATTR-WT: wild-type transthyretin amyloidosis

*V122I; I68L; L111M; T60A; S23N; P24S; W41L; V30M; V20I; APOA1

*** PRKAG2 gene disease, Danon disease (LAMP2)

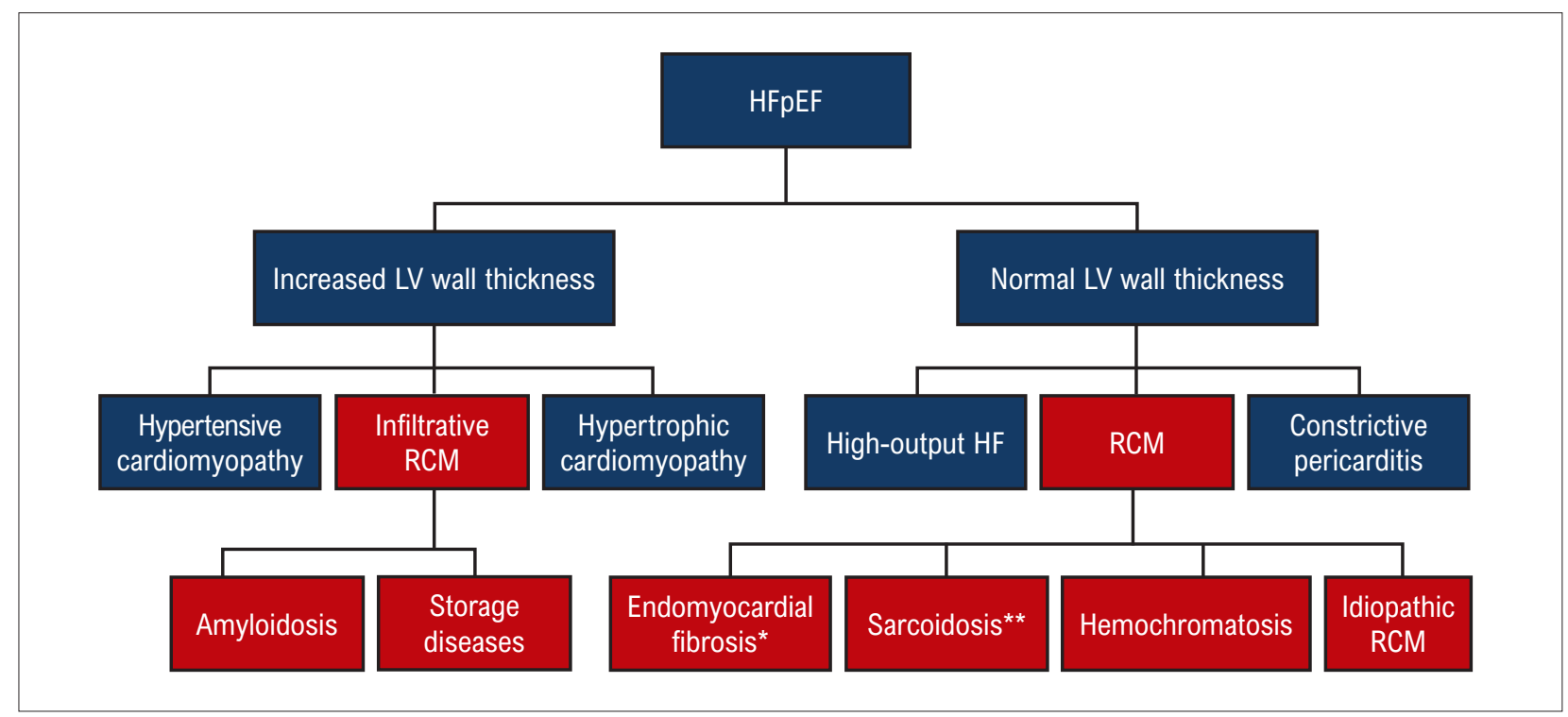

Figure 1 - Differential diagnoses of heart failure with preserved ejection fraction. * Thickening of the left ventricular inferobasal wall and apical obliteration are frequent. ${ }^{* *}$ Abnormal septal thickness (thickening or thinning). HF: heart failure; HFpEF: heart failure with preserved ejection fraction; LV: left ventricle; RCM: restrictive cardiomyopathy. Adapted from Pereira, N.L. et al. J AM Coll Cardiol. 2018; 71 (10)1130-48. 


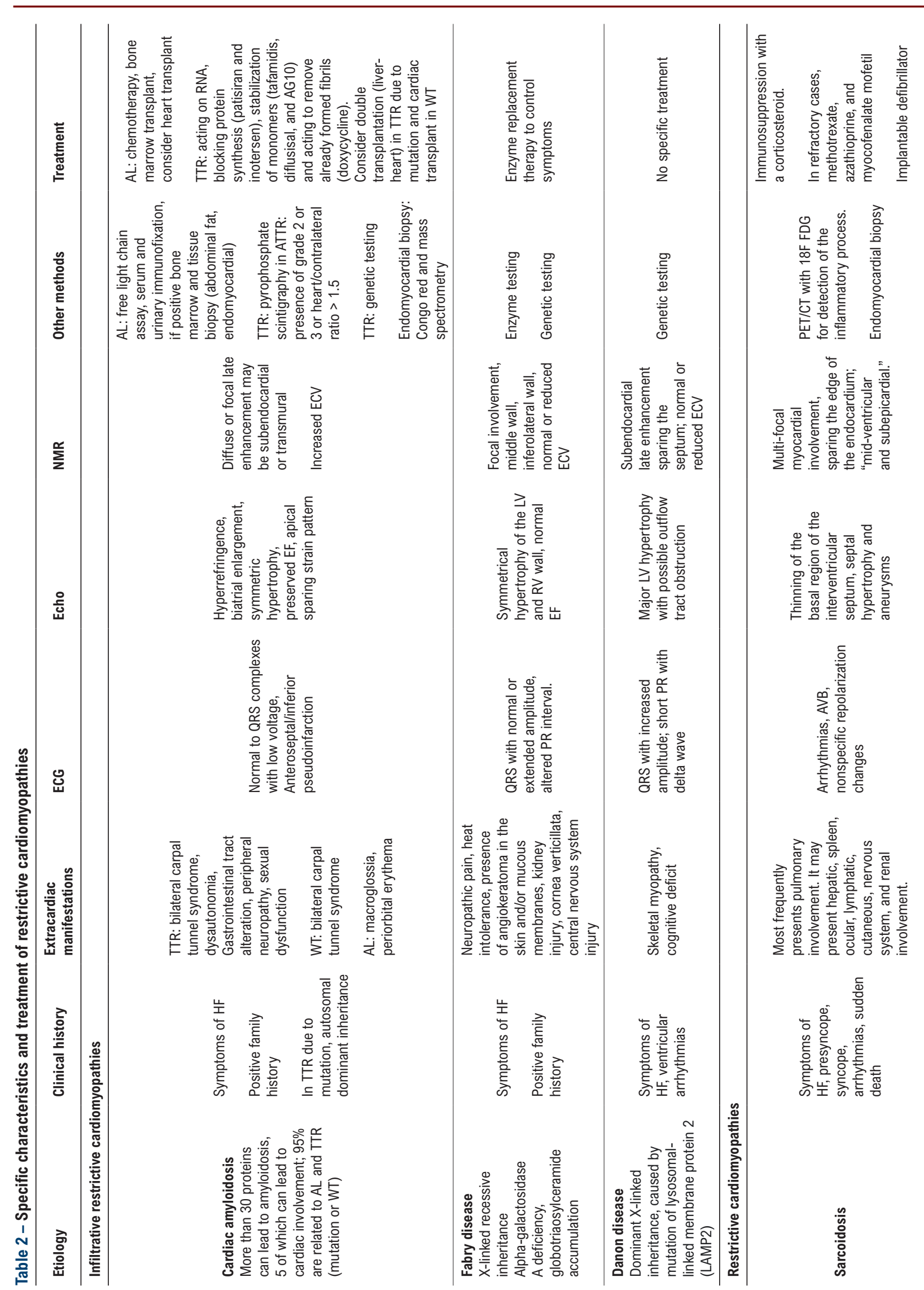




\section{Electrocardiogram}

Electrocardiogram (ECG) is very important to initiate diagnostic reasoning. Infiltrative disorders generate abnormal accumulations of substances in myocytes or in the interstitium of the myocardium, which are responsible for increased wall thickness; therefore, the voltage of the QRS complex may be reduced or incompatible with the degree of thickening in the presence of infiltration between myocardial fibers (a common finding in amyloidosis); on the other hand, when myocardial fibers are involved, we can observe an increase in QRS amplitude on ECG (Fabry disease, for example), with differential diagnoses of diseases related to increased afterload (arterial hypertension and aortic stenosis) and hypertrophic cardiomyopathy. ${ }^{11}$ Although low voltage favors clinical reasoning of cardiac amyloidosis, it is present in only $20 \%$ of patients with the TTR form (transthyretin) and is more frequent in the $\mathrm{AL}$ form ( $50 \%$ of cases). The most common finding related to cardiac amyloidosis is the mismatch between increased ventricular thickness on echocardiogram and the amplitude of the QRS complex; another common finding is the presence of electrically inactive areas in the absence of obstructive coronary artery disease (pseudoinfarction). 7,12 In Fabry disease, ECG abnormalities may include short PR interval, right bundle branch block, left ventricular hypertrophy, and giant negative T waves. ${ }^{13}$

In infiltrative RCM, sinus rhythm with biatrial overload may be observed. Atrial fibrillation, however, is not uncommon. Bundle branch blocks and atrioventricular blocks can also be a presentation of infiltrative diseases. Ventricular arrhythmias are also common. ${ }^{7}$

\section{Echocardiography}

Transthoracic echocardiography typically demonstrates normal ejection fraction, normal chamber volumes with biatrial enlargement, and restrictive diastolic filling parameters. Infiltrative RCM progresses with concentric increase in left ventricular wall and septum thickness; in amyloidosis, we may observe thickening of the right ventricle and interatrial septum. Assessment of left ventricular thickening on transthoracic echocardiography, especially when correlated with ECG, contributes to differential diagnoses in HFpEF (Figure 1). Diastolic compliance is abnormal, and it shows increased early diastolic filling velocity ( $E$ wave), reflecting elevated left atrial pressure, decreased atrial filling velocity (A wave) due to high ventricular diastolic pressures $(E / A>1.5)$, shortening of mitral deceleration time (< $120 \mathrm{~ms})$, and decreased isovolumetric relaxation time. Tissue Doppler imaging reveals reduced initial diastolic longitudinal axis or mitral annular velocities $\left(\mathrm{e}^{\prime}\right)$ and increased $\mathrm{E} / \mathrm{e}^{\prime}$ ratio $(>15)$.

An aspect of myocardial hyperrefringence is often characteristic of amyloid infiltrate. Using the speckle tracking technique, the longitudinal strain pattern can help differentiate between cardiac amyloidosis and hypertrophic cardiomyopathy due to the characteristic of restricted basal movement compared to apical movement in amyloidosis (apical sparing). ${ }^{14-16}$ 


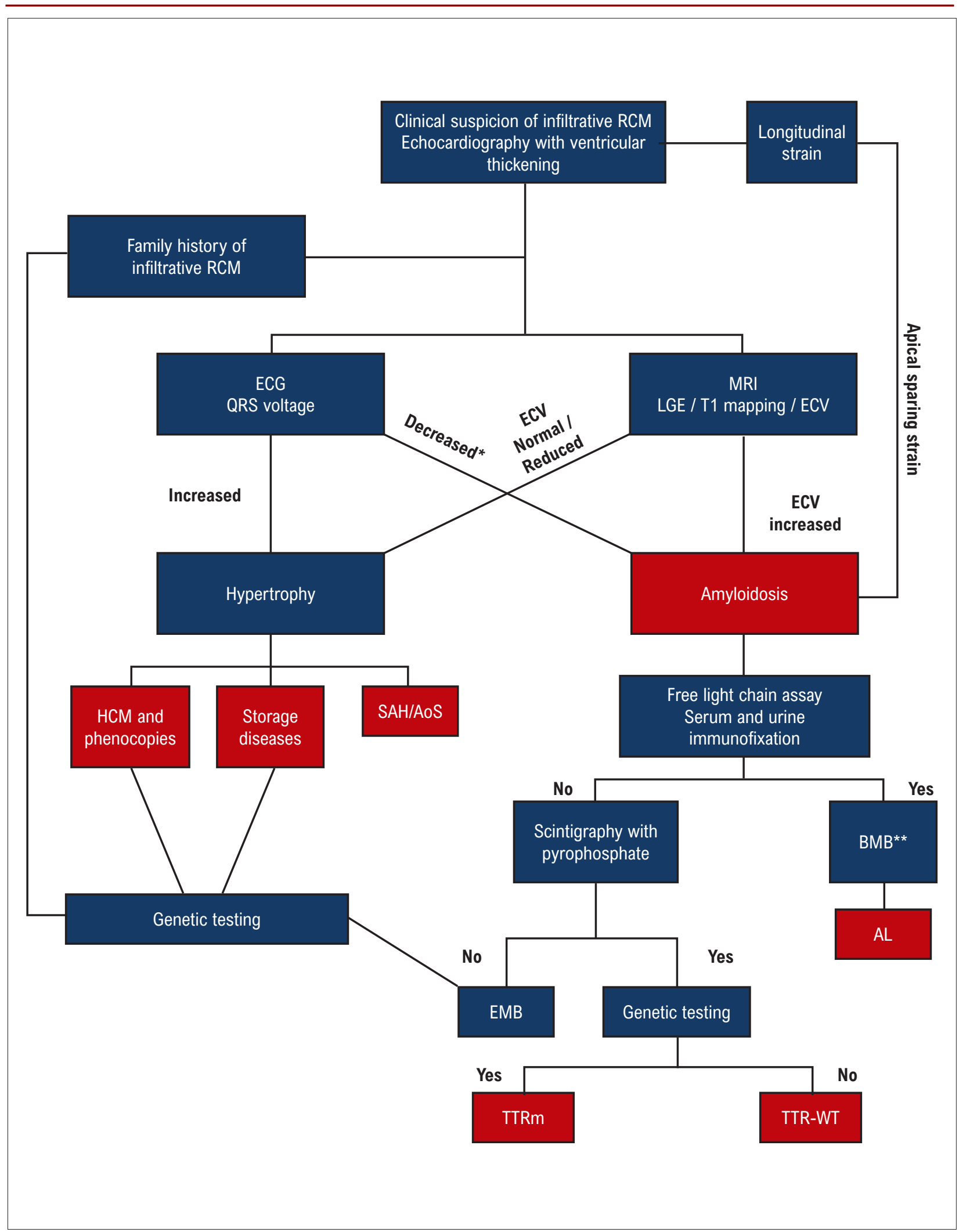

Figure 2 - Flowchart for investigation of restrictive cardiomyopathies with infiltrative phenotypes. * Reduced or normal QRS complex with mismatch between left ventricular wall thickness on echocardiography and QRS complex on electrocardiogram. ** Bone marrow and tissue biopsy of any affected organ (e.g. abdominal fat, endomyocardial). AL: light chain; AoS: aortic stenosis; BMB: bone marrow biopsy; ECG: electrocardiogram; ECV: extracellular volume; EMB: endomyocardial biospy; HCM: hypertrophic cardiomyopathy; LGE: late gadolinium enhancement; MRI: magnetic resonance imaging; SAH: systemic arterial hypertension; TTRm: transthyretin mutation; TTR-WT: transthyretin, wild-type. 


\section{Cardiac resonance}

Cardiac magnetic resonance imaging, with a combination of native and contrast images, allows for accurate anatomical and functional assessment, as well as tissue characterization, indisputably assisting the assessment of RCM. ${ }^{17}$

Late gadolinium enhancement plays an established role in assisting differential diagnosis of cardiomyopathies, including restrictive ones. Diffuse subendocardial late enhancement is indicative of fibrosis, and it is observed in approximately one third of all cases of RCM; however, it is limited to patients with creatinine clearance above $30 \mathrm{~mL} / \mathrm{min}$. Gadolinium is an extracellular contrast agent, and, under normal conditions, it is not retained in the myocardium after administration. Amyloid infiltration results in volume expansion and contrast retention.

One of the greatest advantages of cardiac magnetic resonance imaging is tissue characterization of various structures and cardiac diseases. Recently, this characterization went from being merely qualitative to measuring objectively by means of $\mathrm{T} 1$ and $\mathrm{T} 2$ parametric maps, with the additional advantage of not requiring contrast. T1 mapping has made it possible to objectively measure areas of edema, inflammation, and fibrosis, reflecting systemic changes that occur in the extracellular space. Another advantage of the technique is that it allows measurement of extracellular volume (ECV), with contrast infusion, assisting in differential diagnosis of the restrictive infiltrative cause, given that, in amyloidosis, there is an increase in ECV. On the other hand, in diseases that occur with hypertrophy, there is a more reduced pattern of ECV. ${ }^{18,19}$

\section{Bone tracer scintigraphy}

For suspected amyloidosis, this technique has demonstrated a sensitivity of $99 \%$ and specificity of $86 \%$ for diagnosis of cardiac involvement due to TTR cardiac amyloidosis, whether wild-type or due to a mutation, once the light chain form has been excluded. Radiopharmaceutical uptake is classified by the semiquantitative visual score of cardiac retention in relation to the sternum; the presence of grade 2 or 3 is highly suggestive of TTR in the absence of monoclonal proteins. Furthermore, quantitative analysis of cardiac retention in ratio to the contralateral chest wall greater than 1.5 is consistent for diagnosis of the TTR form. ${ }^{20}$

\section{Endomyocardial biopsy}

The evolution of imaging methods has reduced the need for endomyocardial biopsy in diagnosis, but it may still play a fundamental role in the diagnostic evaluation of patients with restrictive disease. Diagnoses of systemic diseases with cardiac involvement, such as amyloidosis and hemochromatosis, can be definitively established by biopsy of the right ventricle. Endomyocardial biopsy can be used when clinical, laboratory, and imaging data are not sufficient to establish diagnosis. It is worth underscoring that a recent study observed that right ventricular biopsy was effective in only $29 \%$ of patients with unexplained RCM. ${ }^{20}$

\section{Genetic assessment}

The genetic etiology of RCM is strongly suspected, due to its familial occurrence. Clinical and imaging presentations in infiltrative RCM are similar, and they may be difficult to differentiate when the extracardiac clinical expression is not as pronounced; correct genetic diagnosis implies prognostic assessment and specific therapeutic possibilities. In TTR amyloidosis, genetic analysis is essential to differentiate between mutation and wild-type; furthermore, mutational TTR is associated with the phenotypic expression of neuropathy and/or heart disease. It is also important in the differential diagnosis of hypertrophy, including hypertrophic RCM, its phenocopies, and storage diseases. ${ }^{21-23}$

\section{Treatment}

Treatment of RCM must be individualized according to etiology (Table 2). Management of HF is a challenge, given that few pharmacological therapies have been associated with favorable outcomes in this scenario. Patients poorly tolerate antihypertensives and betablockers; digoxin should be avoided due to the risk of intoxication. Diuretic therapy and volume management is the most used strategy. Oral anticoagulation should be considered in the presence of atrial fibrillation, regardless of risk scores, due to the associated high thromboembolic risk. Pacemakers considered in blocks and symptomatic bradycardias and defibrillators are controversial. ${ }^{23-26}$

\section{Conclusion}

HFpEF is currently a great challenge for clinicians, as it represents a phenotype with many possibilities and, in general, limited therapeutic strategies; however, the search for etiologies should not be neglected, as it makes it possible to establish prognosis and specific therapeutic strategies that can modify the evolution of patients. For this purpose, all available workup should be taken into account, from clinical history and detailed physical examination, family history, and involvement of other systems, combined with adequate diagnostic procedures, including ECG, echocardiogram, laboratory tests, nuclear medicine, coronary and hemodynamic assessment, magnetic resonance, and, in specific situations, endomyocardial biopsy and genetic analysis. To paraphrase Claude Bernard, "If you do not know what you are looking for, you will not know how to interpret what you find." Therefore, we must not neglect considering and seeking differential diagnoses of HFpEF, including in RCM with infiltrative phenotypes.

\section{Author Contributions}

Writing of the manuscript and Critical revision of the manuscript for intellectual content: Trevizan LLB, Mangini S 


\section{Potential Conflict of Interest}

No potential conflict of interest relevant to this article was reported.

\section{Sources of Funding}

There were no external funding sources for this study.

\section{Study Association}

This study is not associated with any thesis or dissertation work.

\section{Ethics approval and consent to participate}

This article does not contain any studies with human participants or animals performed by any of the authors.

\section{References}

1. Shah KS, Xu H, Matsouaka RA, Bhatt DL, Heidenreich PA, Hernandez AF, et al. Heart Failure with Preserved, Borderline, and Reduced Ejection Fraction: 5-Year Outcomes. J Am Coll Cardiol. 2017;70(20):2476-86. doi: 10.1016/j. jacc.2017.08.074

2. Benjamin EJ, BlahaMJ, ChiuveSE, Cushman M, Das SR, Deo R, etal. HeartDisease and StrokeStatistics-2017 Update: A Report From theAmerican HeartAssociation. Circulation. 2017;135(10):146-603. doi: 10.1161/CIR.0000000000000485

3. Madan N, Kalra D. Clinical Evaluation of Infiltrative Cardiomyopathies Resulting in Heart Failure with Preserved Ejection Fraction. Rev Cardiovasc Med. 2020;21(2):181-90. doi: 10.31083/j.rcm.2020.02.65.

4. Mesquita ET, Grion DC, Kubrusly MC, Silva BBFF, Santos EAR. Phenotype Mapping of Heart Failure with Preserved Ejection Fraction. Int.J. Cardiovasc. Sci. 2018;31(6):652-61. doi: 10.5935/23594802.20180047.

5. Pereira NL, Grogan M, Dec GW. Spectrum of Restrictive and Infiltrative Cardiomyopathies: Part 1 of a 2-Part Series. J Am Coll Cardiol. 2018;71(10):1130-48. doi: 10.1016/j.jacc.2018.01.016

6. Muchtar E, Blauwet LA, Gertz MA. Restrictive Cardiomyopathy: Genetics, Pathogenesis, Clinical Manifestations, Diagnosis, and Therapy. Circ Res. 2017;121(7):819-37. doi: 10.1161/CIRCRESAHA.117.310982.

7. Seward JB, Casaclang-Verzosa G. Infiltrative Cardiovascular Diseases: Cardiomyopathies That Look Alike. J Am Coll Cardiol. 2010;55(17):176979. doi: 10.1016/j.jacc.2009.12.040

8. Ammash NM, Seward JB, Bailey KR, Edwards WD, Tajik AJ. Clinical Profile and Outcome of Idiopathic Restrictive Cardiomyopathy. Circulation. 2000;101(21):2490-6. doi: 10.1161/01.cir.101.21.2490.

9. Pfeffer MA, Shah AM, Borlaug BA. Heart Failure with Preserved Ejection Fraction In Perspective. Circ Res. 2019;124(11):1598-617. doi: 10.1161/ CIRCRESAHA.119.313572.

10. Hausfater P, Costedoat-Chalumeau N, Amoura Z, Cacoub P, Papo T, Grateau G, et al. AL Cardiac Amyloidosis and Arterial Thromboembolic Events. Scand J Rheumatol. 2005;34(4):315-9. doi: 10.1080/03009740510015203.

11. Maurer MS, Elliott P, Comenzo R, Semigran M, Rapezzi C. Addressing Common Questions Encountered in the Diagnosis and Management of Cardiac Amyloidosis. Circulation. 2017;135(14):1357-77. doi: 10.1161/ CIRCULATIONAHA.116.024438

12. Cyrille NB, Goldsmith J, Alvarez J, Maurer MS. Prevalence and Prognostic Significance of Low QRS Voltage Among the Three Main Types of Cardiac Amyloidosis. Am J Cardiol. 2014;114(7):1089-93. doi: 10.1016/j. amjcard.2014.07.026.

13. Weidemann F, Reiser M. Fabry Disease: Cardiomyopathy Staging. JACC Cardiovasc Imaging. 2019;12(8):1684-5. doi: 10.1016/j.jcmg.2018.05.029.
14. Stricagnoli M, Cameli M, Incampo E, Lunghetti S, Mondillo S. Speckle Tracking Echocardiography in Cardiac Amyloidosis. Heart Fail Rev. 2019;24(5):701-7. doi: 10.1007/s10741-019-09796-z.

15. Bellavia D, Pellikka PA, Al-Zahrani GB, Abraham TP, Dispenzieri A Miyazaki C, et al. Independent Predictors of Survival in Primary Systemic (Al) Amyloidosis, Including Cardiac Biomarkers and Left Ventricular Strain Imaging: An Observational Cohort Study. J Am Soc Echocardiogr. 2010;23(6):643-52. doi: 10.1016/j.echo.2010.03.027.

16. Koyama J, Falk RH. Prognostic Significance of Strain Doppler Imaging in Light-Chain Amyloidosis. JACC Cardiovasc Imaging. 2010;3(4):333-42. doi: 10.1016/j.jcmg.2009.11.013

17. Galea N, Polizzi G, Gatti M, Cundari G, Figuera M, Faletti R. Cardiovascula Magnetic Resonance (CMR) in Restrictive Cardiomyopathies. Radiol Med. 2020;125(11):1072-86. doi: 10.1007/s11547-020-01287-8.

18. Abulizi M, Sifaoui I, Wuliya-Gariepy M, Kharoubi M, Israël JM, Emsen B, et al. 18F-Sodium Fluoride PET/MRI Myocardial Imaging in Patients with Suspected Cardiac Amyloidosis. J Nucl Cardiol. 2021;28(4):1586-95. doi: 10.1007/s12350-019-01885-8.

19. Martinez-Naharro A, Treibel TA, Abdel-Gadir A, Bulluck H, Zumbo G, Knight DS, et al. Magnetic Resonance in Transthyretin Cardiac Amyloidosis. J Am Coll Cardiol. 2017;70(4):466-77. doi: 10.1016/j.jacc.2017.05.053.

20. Gillmore JD, Maurer MS, Falk RH, Merlini G, Damy T, Dispenzieri A, et al Nonbiopsy Diagnosis of Cardiac Transthyretin Amyloidosis. Circulation. 2016;133(24):2404-12. doi: 10.1161/CIRCULATIONAHA.116.021612.

21. Chung WK. Predictive Genetic Testing for Cardiomyopathies. Prog Pediat Cardiol. 2007;23(1):33-8. doi: 10.1016/j.ppedcard.2007.05.002.

22. Colombo MG, Botto N, Vittorini S, Paradossi U, Andreassi MG. Clinical Utility of Genetic Tests for Inherited Hypertrophic and Dilated Cardiomyopathies. Cardiovasc Ultrasound. 2008;6:62. doi: 10.1186/1476-7120-6-62.

23. Hershberger RE, Givertz MM, Ho CY, Judge DP, Kantor PF, McBride KL, et al Genetic Evaluation of Cardiomyopathy-A Heart Failure Society of America Practice Guideline. J Card Fail. 2018;24(5):281-302. doi: 10.1016/j. cardfail.2018.03.004

24. Maurer MS, SchwartzJH, Gundapaneni B, Elliott PM, Merlini G, WaddingtonCruz M, et al. Tafamidis Treatment for Patients with Transthyretin Amyloid Cardiomyopathy. N Engl J Med. 2018;379(11):1007-16. doi: 10.1056/ NEJMoa1805689.

25. Ribeiro Neto ML, Jellis CL, Joyce E, Callahan TD, Hachamovitch R, Culve DA. Update in Cardiac Sarcoidosis. Ann Am Thorac Soc. 2019;16(11):134150. doi: 10.1513/AnnalsATS.201902-119CME.

26. Azevedo O, Gago MF, Miltenberger-Miltenyi G, Sousa N, Cunha D. Fabry Disease Therapy: State-of-the-Art and Current Challenges. Int J Mol Sci. 2020;22(1):206. doi: 10.3390/ijms22010206. 